\title{
Probability of Error Analysis of BPSK OFDM Systems with Random Residual Frequency Offset
}

\author{
P. C. Weeraddana, Nandana Rajatheva, Senior Member, IEEE, and H. Minn, Senior Member, IEEE
}

\begin{abstract}
In this paper, we derive closed form bit error rate (BER) expressions for orthogonal frequency division multiplexing (OFDM) systems with residual carrier frequency offset (CFO). Most of the published work treats CFO as a nonrandom parameter. But in our study we consider it as a random parameter. The BER performance of binary phase shift keying (BPSK) OFDM system is analyzed in the cases of additive white Gaussian noise (AWGN), frequency-flat and frequency-selective Rayleigh fading channels. We further discuss how these expressions can be related to systems with practical estimators. The simulation results are provided to verify the accuracy of these error rate expressions.
\end{abstract}

Index Terms-Probability density function (pdf), Frequency offset, Inter-carrier interference, Frequency-selective fading, Frequency-flat fading, Cramer-Rao Lower Bound (CRB).

\section{INTRODUCTION}

$\mathbf{O}$ RTHOGONAL Frequency Division Multiplexing (OFDM) is a bandwidth efficient signaling scheme where the orthogonality among the subcarriers should be maintained to a high degree of precision. Since the spectra of the sub-carriers are overlapping, an accurate frequency synchronization technique is needed. However, due to oscillator inaccuracies and non-ideal receiver synchronization, the orthogonality of subcarriers is compromised resulting in intercarrier interference (ICI) which can degrade the performance of OFDM systems significantly [1].

There are two different approaches to addressing the ICI problem induced by the carrier frequency offset (CFO). The first approach performs CFO estimation and compensation. There exist several CFO estimation techniques which can be categorized as training based methods [2]-[7] and semiblind or blind methods [8][9]. The training based methods offer faster synchronization, lower complexity, and more reliable performance at the cost of training overhead while the semi-blind or blind methods save training overhead at the expense of longer latency, higher complexity, and less reliable performance. The second approach applies a self ICI cancellation [10][11] at the sacrifice of data rate. In all current OFDM systems, the first approach is adopted. After the CFO

Paper approved by C. Tepedelenlioglu, the Editor for Synchronization and Equalization. Manuscript received April 27, 2007; revised September 21, 2007, January 7, 2008, and January 16, 2008.

P.C. Weeraddana and N. Rajatheva are with the Telecommunications Field of Study, School of Engineering Technology, Asian Institute of Technology, P.O. Box 4, Klong Luang 12120, Pathumthani, Thailand (e-mail: rajath@ait.ac.th).

H. Minn is with the Department of Electrical Engineering, University of Texas at Dallas, P.O.Box 830688, EC 33, Richardson, TX 75083-0688, U.S.A (e-mail: hlaing.minn@utdallas.edu).

This paper was presented in part at the 2007 IEEE 65th Vehicular Technology Conference VTC2007-Spring, Dublin, Ireland, April 22-25, 2007.

Digital Object Identifier 10.1109/TCOMM.2009.060683 estimation and compensation, there still exists a residual CFO which affects the system error performance.

The bit error rate (BER) analysis of OFDM in the presence of CFO has recently attracted significant research attention [12]-[20] due to the increased popularity of OFDM and its high sensitivity to CFO. The existing works in [12][13] adopted an approach which treats ICI as a Gaussian process based on the central limit theorem. However, this approach does not yield satisfactory results at high signal to noise ratios (SNR) [14]. In contrast, the approach followed in [15] uses the characteristic function and Beaulieu series to derive exact BER expressions for additive white Gaussian noise (AWGN) channel in the presence of ICI where the probability of error is expressed conditioned on the normalized CFO. In [16][17], the authors have derived exact BER/SER expressions for AWGN, frequency-flat and frequency-selective channels with fixed CFO error. The authors in [18][19] also provide some error performance studies with fixed CFO.

All of the above existing works address error performance affected by a fixed CFO. In practice, the CFO affecting the average error performance is a random variable (This will be elaborated in Section III). Hence, in this paper we consider the BER performance affected by a random CFO. To the best of our knowledge, only [21][22] considered random $\mathrm{CFO}$ in error performance analysis, but the CFO affecting the error performance was assumed to be independent of the channel and the ICI term was treated as a colored complexed Gaussian process. If CFO estimation and compensation are performed at the receiver as in typical wireless systems, the (residual) CFO does depend on the channel. Furthermore, treating ICI as Gaussian is just an approximation and can give unsatisfactory results [14]. Hence, in contrast to the existing work with random CFO, in this paper we do not limit to the channel-independent CFO case and do not assume Gaussian ICI approximation.

In this paper we present the BER analysis of binary phase shift keying (BPSK) OFDM systems with a random CFO. Our technical contributions are summarized below. We present a new approach in contrast to the Gaussian ICI approximation based approaches of the existing work. We consider random $\mathrm{CFO}$ while most existing works addressed deterministic CFO only. We cover several random $\mathrm{CFO}$ scenarios (e.g., channeldependent/independent $\mathrm{CFO}$, with or without $\mathrm{CFO}$ estimation, etc.) while existing works did not.

The rest of the paper is organized as follows. In Section II we present the system model and introduce a novel approximation to the ICI coefficients. In Section III we derive closed form BER expressions for AWGN, frequency-flat and 
frequency-selective Rayleigh fading channels with a channelindependent uniformly-distributed (residual) CFO. Section IV addresses the channel-dependent $\mathrm{CFO}$ case where we obtain the BER expression for a frequency-flat Rayleigh fading channel with a random residual $\mathrm{CFO}$ which, conditioned on the channel, is Gaussian-distributed. An alternative approach with more relaxed assumptions is presented in Section $\mathrm{V}$ for the BER analysis with a random (residual) CFO in both frequency-flat and selective Rayleigh fading channels. The BER analyses in Section III and IV assume that the perfect channel knowledge is available at the receiver, while Section $\mathrm{V}$ includes channel estimation errors in the analysis. Section VI provides simulation results to verify our theoretical results. Finally, Section VII concludes the paper.

Notations: $(.)^{H},(.)^{T}$, and $\overline{(.)}$ denote the Hermitian transpose, the transpose, and the conjugate operations, respectively. $\mathbf{1}_{k}$ and $\mathbf{0}_{k}$ represent the all-one column vector and the allzero column vector of length $k$ while $\boldsymbol{I}_{k}$ and $\mathbf{0}_{k \times n}$ denote the $k \times k$ identity matrix and the $k \times n$ all-zero matrix, respectively. The $N$-point unitary discrete Fourier transform (DFT) matrix is denoted by $\boldsymbol{F}=\left[\boldsymbol{f}_{0} \boldsymbol{f}_{1} \ldots \boldsymbol{f}_{N-1}\right]$ where $\boldsymbol{f}_{k}=\left[1 e^{-j 2 \pi k / N} \cdots e^{-j 2 \pi k(N-1) / N}\right]^{T} / \sqrt{N}$. We define $\boldsymbol{F}_{L}=$ $\left[\boldsymbol{f}_{0} \boldsymbol{f}_{1} \cdots \boldsymbol{f}_{L-1}\right] .[\boldsymbol{X}]_{k, n}$ represents the $(k, n)$ th element of the matrix $\boldsymbol{X}$, and $\operatorname{diag}\{\boldsymbol{x}\}$ denotes a diagonal matrix with diagonal elements defined by $\boldsymbol{x} . \Re(\boldsymbol{x})$ denotes the real part of x. $E\{$.$\} represents the statistical expectation.$

\section{System Model AND ICI COEFFICIENTS}

We first present the signal model for a frequency-selective fading channel from which the models for AWGN and frequency-flat fading channels can easily be obtained. We consider an OFDM system with $N$ subcarriers in a quasi-static channel. In the presence of a normalized (by the subcarrier spacing) CFO $v$, the time-domain received signal vector after the cyclic prefix removal is given by [7]

$$
\boldsymbol{r}=\boldsymbol{\Gamma}(v) \boldsymbol{S h}+\boldsymbol{w}=\sqrt{N} \boldsymbol{\Gamma}(v) \boldsymbol{F}^{H} \boldsymbol{H} \boldsymbol{c}+\boldsymbol{w}
$$

where $\boldsymbol{r}=\left[\begin{array}{llll}r_{0} & r_{1} & \cdots & r_{N-1}\end{array}\right]^{T}, \boldsymbol{c}=\left[\begin{array}{llll}c_{0} & c_{1} & \cdots & c_{N-1}\end{array}\right]^{T}$, $\boldsymbol{h}=\left[\begin{array}{llll}h_{0} & h_{1} & \cdots & h_{L-1}\end{array}\right]^{T}, \boldsymbol{w}=\left[\begin{array}{llll}w_{0} & w_{1} & \cdots & w_{N-1}\end{array}\right]^{T}$, $\boldsymbol{\Gamma}(v)=\operatorname{diag}\left[\begin{array}{llll}1 & e^{j 2 \pi v / N} & \cdots & e^{j 2 \pi(N-1) v / N}\end{array}\right]$ and $\boldsymbol{H}=$ $\operatorname{diag}\left\{\boldsymbol{F}_{\boldsymbol{L}} \boldsymbol{h}\right\}=\operatorname{diag}\left[\begin{array}{llll}H_{0} & H_{1} & \cdots & H_{N-1}\end{array}\right]^{T}$. Here $\left\{h_{n}\right\}$ denote the channel impulse response (CIR) coefficients and $L$ is the number of CIR taps. $\left\{w_{n}\right\}$ are independent and identically distributed zero-mean circularly-symmetric complex Gaussian noise samples each having a variance of $\sigma^{2}$ per dimension. $\left\{c_{n}\right\}$ are independent equi-probable frequency domain transmit symbols and the corresponding time-domain signal vector is given by $\boldsymbol{s}=\left[\begin{array}{llll}s_{0} & s_{1} & \cdots & s_{N-1}\end{array}\right]^{T}=\boldsymbol{F}^{H} \boldsymbol{c}$. The time-domain signal matrix in (1) is defined by $[\boldsymbol{S}]_{k, n}=s_{k-n}, 0 \leq k \leq$ $N-1,0 \leq n \leq L-1$ with $s_{k}=\frac{1}{\sqrt{N}} \sum_{n=0}^{N-1} c_{n} e^{j 2 \pi n k / N}$ for $k=-L+1, \cdots, N-1$.

Let $\hat{v}$ be the estimated frequency offset, $\hat{v}=v+v_{\Delta}$ where we denote the residual CFO as $v_{\Delta}$. After the frequency offset compensation and DFT, the received signal vector is given by

$$
\boldsymbol{R}=\sqrt{N} \boldsymbol{F} \boldsymbol{\Gamma}^{H}\left(v_{\Delta}\right) \boldsymbol{F}^{H} \boldsymbol{H} \boldsymbol{c}+\boldsymbol{w}^{\prime}
$$

where $\boldsymbol{R}=\left[\begin{array}{llll}R_{0} & R_{1} & \cdots & R_{N-1}\end{array}\right]^{T}$ and $\boldsymbol{w}^{\prime}=\left[\begin{array}{llll}n_{0}^{\prime} & n_{1}^{\prime} & \cdots & n_{N-1}^{\prime}\end{array}\right]^{T} . \boldsymbol{w}^{\prime}$ has the same statistical properties as $\boldsymbol{w}$. Evaluating the $(k, l)$ th element of $\boldsymbol{F} \boldsymbol{\Gamma}^{H}\left(v_{\Delta}\right) \boldsymbol{F}^{H}$ and denoting it as $I_{l-k}^{\prime}$ (ICI coefficient), we can obtain

$$
I_{l-k}^{\prime}=\frac{1}{N} \sum_{n=0}^{N-1} \exp \left\{j 2 \pi\left(l-k-v_{\Delta}\right) \frac{n}{N}\right\} ; \quad k, l \in \mathcal{K} .
$$

where $\mathcal{K}=\{0,1, \cdots, N-1\}$. Then, using (2), we can express the received symbol on the $k$ th subcarrier as

$$
R_{k}=\sqrt{N} c_{k} H_{k} I_{0}^{\prime}+\sqrt{N} \sum_{l=0, l \neq k}^{N-1} c_{l} H_{l} I_{l-k}^{\prime}+n_{k}^{\prime} ; \quad k \in \mathcal{K} .
$$

The expressions for AWGN and frequency-flat fading channels can be derived using (4).

Next, we analyze the ICI coefficients. With the assumption that the residual CFO is very small and using the approximation $\exp (j x) \simeq(1+j x)$ (this approximation is extensively used in the literature in OFDM system analysis for very small values of the phase noise [23][24][25]) for small real-valued $x$, we get

$$
\begin{aligned}
I_{l-k}^{\prime} \approx \frac{1}{N} \sum_{n=0}^{N-1} & \exp \left\{j 2 \pi(l-k) \frac{n}{N}\right\} \\
& \quad-j v_{\Delta} \frac{2 \pi}{N^{2}} \sum_{n=0}^{N-1} n \cdot \exp \left\{j 2 \pi(l-k) \frac{n}{N}\right\} .
\end{aligned}
$$

With some trigonometric manipulations [25,eq. 1.352], we can further reduce the above relation as (see Appendix for details)

$$
I_{l-k}^{\prime} \approx \begin{cases}\frac{\pi v_{\Delta}}{N}\left[-\cot \left(\frac{\pi(l-k)}{N}\right)+j\right] & , \text { if } l \neq k \\ 1-j \pi \frac{N-1}{N} v_{\Delta} & , \text { if } l=k\end{cases}
$$

We will use (6) in our BER analysis since the residual CFO is typically small in practical OFDM systems.

\section{Performance Analysis with CHANNEL-INDEPENDENT (RESIDUAL) CFO}

We can treat the CFO and the CIR as independent parameters under some conditions. An example is a scenario where the transceivers use highly-stable crystal oscillators and skip CFO estimation to save energy. Although a crystal oscillator provides a stable frequency, different oscillators (of different users) may have slightly different frequencies which can also depend on their temperatures. Our interest is the (ensemble average) BER of a user (not a particular user with a particular CFO). Under this condition CFO could be modeled as Gaussian. Alternatively, as a worst case design, we may consider the $\mathrm{CFO}$ (not the residual $\mathrm{CFO}$ ) to be uniformly distributed and independent of the channel. Uniform random CFO is often used in the literature (e.g;[5]). Another example is a scenario where the receiver performs CFO estimation and compensation. Under this scenario the residual CFO can be treated as a Gaussian random variable (by invoking the asymptotic property of maximum likelihood (ML) estimation) which is independent of the channel (see Section III-D) in a system with perfect power control and dependent on the channel if without power control. 
For the channel-independent residual CFO case, BER is obtained by solving the following

$$
P_{b}(\xi)=\iint P_{b}\left(\xi \mid v_{\Delta}, \boldsymbol{h}\right) f\left(v_{\Delta}\right) f(\boldsymbol{h}) d v_{\Delta} d \boldsymbol{h}
$$

where $f\left(v_{\Delta}\right)$ and $f(\boldsymbol{h})$ are pdfs of residual CFO and channel respectively, and $P_{b}\left(\xi \mid v_{\Delta}, \boldsymbol{h}\right)$ represents the BER conditioned on $v_{\Delta}$ and $\boldsymbol{h}$. In the following, the $v_{\Delta}$ in the BER expression will represent the CFO for the scenario without CFO estimation, and the residual CFO for the scenario with $\mathrm{CFO}$ estimation and compensation. In the following sections A, B and $\mathrm{C}$, we consider the uniformly distributed CFO (without CFO estimation), while in Section D we address the scenario with $\mathrm{CFO}$ estimation (i.e., a Gaussian-distributed residual CFO).

\section{A. AWGN Channel with Uniformly Distributed CFO}

For the AWGN channel, we have $\boldsymbol{H}=\frac{1}{\sqrt{N}} \boldsymbol{I}_{N}$. We can simply deduce from (4) that

$$
R_{k}=c_{k} I_{0}^{\prime}+\sum_{l=0, l \neq k}^{N-1} c_{l} I_{l-k}^{\prime}+n_{k}^{\prime} ; \quad k=0,1, \cdots, N-1 .
$$

Following [16], we obtain the characteristic function (CHF) of the real part of $R_{0}, \Re\left(R_{0}\right)$, as

$$
\begin{aligned}
& \phi_{\Re\left(R_{0}\right)}(\omega)=\frac{1}{2^{N-1}} \sum_{k=1}^{2^{N-2}}( \exp \left\{j \omega \theta_{k}-\frac{\omega^{2} \sigma^{2}}{2}\right\} \\
&\left.+\exp \left\{j \omega \beta_{k}-\frac{\omega^{2} \sigma^{2}}{2}\right\}\right)
\end{aligned}
$$

where $\theta_{k}=\left(1+a_{k} v_{\Delta}\right), \quad \beta_{k}=\left(1-a_{k} v_{\Delta}\right), \quad a_{k}=\boldsymbol{P}^{T} \boldsymbol{e}_{k}$, $\boldsymbol{P}=\frac{\pi}{N}\left[\cot \left(\frac{\pi}{N}\right) \cot \left(\frac{2 \pi}{N}\right) \ldots \cot \left(\frac{(N-1) \pi}{N}\right)\right]^{T}, \boldsymbol{e}_{k}$ is the binary representation of the number $2^{M}-k$ where zeros are replaced with -1 s [16]. Here (9) represents the CHF of a mixture of Gaussian density functions and for BPSK signal constellation we can write the conditional bit error probability as [16]

$$
\begin{aligned}
P_{b}\left(\xi \mid v_{\Delta}\right)=\frac{1}{2^{N-1}} \sum_{k=1}^{2^{N-2}}\{ & Q\left(\sqrt{2 \gamma}\left(1+a_{k} v_{\Delta}\right)\right) \\
& \left.+Q\left(\sqrt{2 \gamma}\left(1-a_{k} v_{\Delta}\right)\right)\right\}
\end{aligned}
$$

where $Q(x)$ is the Gaussian Q-function. Then with the assumption that $v_{\Delta}$ is uniformly distributed over the region $[-b, b]$, the BER can be written as

$$
P_{b}(\xi)=\frac{1}{2 b} \int_{-b}^{b} P_{b}\left(\xi \mid v_{\Delta}\right) d v_{\Delta} .
$$

After some algebraic manipulations, (10), (11) gives the bit error probability which is given in (12), where $\lambda_{k}=\left(1+a_{k} b\right)$, $\mu_{k}=\left(1-a_{k} b\right), \gamma=\frac{E_{b}}{N_{0}}=\frac{1}{2 \sigma^{2}}$.

\section{B. Frequency-Flat Rayleigh Fading Channel with Uniformly Distributed $C F O$}

In the frequency-flat Rayleigh fading case, we have $\boldsymbol{H}=$ $(\alpha / \sqrt{N}) \boldsymbol{I}_{N}$ where $\alpha$ is a zero-mean circularly-symmetric complex Gaussian random variable with a variance of $\sigma_{R}^{2}$ per dimension which was taken to be 0.5 . Then, (4) becomes

$$
R_{k}=\alpha c_{k} I_{0}^{\prime}+\alpha \sum_{l=0, l \neq k}^{N-1} c_{l} I_{l-k}^{\prime}+n_{k}^{\prime} ; \quad k=0,1, . ., N-1 .
$$

Then the conditional bit error probability can be written as

$$
\begin{aligned}
P_{b}\left(\xi \mid v_{\Delta}, \alpha\right)=\frac{1}{2^{N-1}} & \sum_{k=1}^{2^{N-2}}\left\{Q\left(\sqrt{2 \gamma}|\alpha|\left(1+a_{k} v_{\Delta}\right)\right)\right. \\
& \left.+Q\left(\sqrt{2 \gamma}|\alpha|\left(1-a_{k} v_{\Delta}\right)\right)\right\} .
\end{aligned}
$$

Note that $|\alpha|$ is Rayleigh distributed with its pdf given by

$$
f(|\alpha|)=\frac{|\alpha|}{\sigma_{R}^{2}} \exp \left(-\frac{|\alpha|^{2}}{2 \sigma_{R}^{2}}\right) .
$$

Averaging (14) over $v_{\Delta}$ and $\alpha$, and after some algebraic manipulations, we obtain the bit error probability as given in (16).

\section{Frequency-Selective Rayleigh Fading Channel with Uni- formly Distributed CFO}

In the case of a frequency-selective channel, the received symbol on the $k$ th subcarrier is given by (4). We assume an $L$ sample-spaced tap-delay-line model for the channel with

$$
\begin{aligned}
& P_{b}(\xi)=\frac{1}{b 2^{N-1}}\left[\sum_{k=1, a_{k} \neq 0}^{2^{N-2}}\left\{\frac{\lambda_{k}}{a_{k}} Q\left(\sqrt{2 \gamma} \lambda_{k}\right)-\frac{\mu_{k}}{a_{k}} Q\left(\sqrt{2 \gamma} \mu_{k}\right)\right\}\right. \\
&\left.+\sum_{k=1, a_{k} \neq 0}^{2^{N-2}}\left\{\frac{-1}{2 \sqrt{\pi \gamma} a_{k}} e^{-\gamma \lambda_{k}^{2}}+\frac{1}{2 \sqrt{\pi \gamma} a_{k}} e^{-\gamma \mu_{k}^{2}}\right\}+\sum_{k=1, a_{k}=0}^{2^{N-2}}\{2 b Q(\sqrt{2 \gamma})\}\right] \\
& P_{b}(\xi)=\frac{1}{2^{N-1} b} \sum_{k=1, a_{k} \neq 0}^{2^{N-2}}\left\{\frac{\lambda_{k}}{2 a_{k}}\left(1-\frac{\sqrt{2 \gamma} \sigma_{R} \lambda_{k}}{\sqrt{1+2 \gamma \sigma_{R}^{2} \lambda_{k}^{2}}}\right)-\frac{\mu_{k}}{2 a_{k}}\left(1-\frac{\sqrt{2 \gamma} \sigma_{R} \mu_{k}}{\sqrt{1+2 \gamma \sigma_{R}^{2} \mu_{k}^{2}}}\right)\right. \\
&\left.+\frac{-1}{2 \sqrt{2 \gamma \sigma_{R}^{2}} a_{k} \sqrt{1+2 \gamma \lambda_{k}^{2} \sigma_{R}^{2}}}+\frac{1}{2 \sqrt{2 \gamma \sigma_{R}^{2}} a_{k} \sqrt{1+2 \gamma \mu_{k}^{2} \sigma_{R}^{2}}}\right\}+\frac{1}{2^{N-1}} \sum_{k=1, a_{k}=0}^{2^{N-2}}\left(1-\sqrt{\frac{2 \sigma_{R}^{2} \gamma}{1+2 \sigma_{R}^{2} \gamma}}\right)
\end{aligned}
$$


the time domain tap coefficients $\left\{h_{l}, l=0,1, \cdots, L-1\right\}$ modeled as zero mean circularly symmetric complex Gaussian random variables having variances $\left\{\sigma_{h_{l}}^{2}\right\}$ with $\sigma_{h_{0}}^{2}+\sigma_{h_{1}}^{2}+$ $\cdots+\sigma_{h_{L-1}}^{2}=1$. Define $\alpha_{l}=\sqrt{N} H_{l}$ for $l=0,1, \cdots, N-1$, $\boldsymbol{\alpha}=\left[\begin{array}{llll}\alpha_{1} & \alpha_{2} & \cdots & \alpha_{N-1}\end{array}\right]^{T}$, and $\sigma_{n}^{2}=\left|\alpha_{0}\right|^{2} \sigma^{2}$. Following the same set of arguments as in [17] we can obtain the conditional $\mathrm{CHF}$ of the random variable $\Re\left(R_{0}\right) \mid \alpha_{0}, \boldsymbol{\alpha}, v_{\Delta}$ as

$$
\begin{array}{r}
\phi_{\Re\left(R_{0}\right) \mid \alpha_{0}, \boldsymbol{\alpha}, v_{\Delta}}(\omega)=\frac{1}{2^{N-1}} \sum_{k=1}^{2^{N-2}}\left(\exp \left\{j \omega \theta_{k}-\frac{\omega^{2} \sigma_{n}^{2}}{2}\right\}\right. \\
\left.+\exp \left\{j \omega \beta_{k}-\frac{\omega^{2} \sigma_{n}^{2}}{2}\right\}\right)
\end{array}
$$

where $\theta_{k}=\left(\left|\alpha_{0}\right|^{2}+\Re\left(\bar{\alpha}_{0} \boldsymbol{P}_{k}^{T} \boldsymbol{\alpha}\right)\right), \beta_{k}=\left(\left|\alpha_{0}\right|^{2}-\Re\left(\bar{\alpha}_{0} \boldsymbol{P}_{k}^{T} \boldsymbol{\alpha}\right)\right)$, and $\boldsymbol{P}_{k}=\operatorname{diag}\left(I_{1}^{\prime}, I_{2}^{\prime}, \ldots, I_{N-1}^{\prime}\right) \boldsymbol{e}_{k}$. Hence, the conditional BER can be obtained as

$$
\begin{aligned}
P_{b}\left(\xi \mid \alpha_{0}, \boldsymbol{\alpha}, v_{\Delta}\right)= & \frac{1}{2^{N-1}} \sum_{k=1}^{2^{N-2}}\left\{Q\left(\frac{\left|\alpha_{0}\right|^{2}+\Re\left(\bar{\alpha}_{0} \boldsymbol{P}_{k}^{T} \boldsymbol{\alpha}\right)}{\sigma_{n}}\right)\right. \\
& \left.+Q\left(\frac{\left|\alpha_{0}\right|^{2}-\Re\left(\bar{\alpha}_{0} \boldsymbol{P}_{k}^{T} \boldsymbol{\alpha}\right)}{\sigma_{n}}\right)\right\} . \quad(18)
\end{aligned}
$$

Define $z_{k}=\Re\left(\bar{\alpha}_{0} \boldsymbol{P}_{k}^{T} \boldsymbol{\alpha}\right)$. It is obvious that the conditional random variable $z_{k} \mid \alpha_{0}, v_{\Delta}$ is Gaussian with mean and variance to be determined. Now we have the following [27]:

$$
\begin{gathered}
\boldsymbol{C}=E\left\{\left(\alpha_{0} \boldsymbol{\alpha}^{T}\right)^{T}\left(\bar{\alpha}_{0} \boldsymbol{\alpha}^{H}\right)\right\}=\left(\begin{array}{cc}
c_{\alpha_{0} \alpha_{0}} & \boldsymbol{C}_{\boldsymbol{\alpha} \alpha_{0}}^{H} \\
\boldsymbol{C}_{\boldsymbol{\alpha} \alpha_{0}} & \boldsymbol{C}_{\boldsymbol{\alpha} \boldsymbol{\alpha}}
\end{array}\right) \\
=N \boldsymbol{F}_{L} \boldsymbol{C}_{h} \boldsymbol{F}_{L}^{H} \\
E\left\{\boldsymbol{\alpha} \mid \alpha_{0}\right\}=\alpha_{0} c_{\alpha_{0} \alpha_{0}}^{-1} \boldsymbol{C}_{\boldsymbol{\alpha} \alpha_{0}} \\
\boldsymbol{C}_{\boldsymbol{\alpha} \mid \alpha_{0}}=\boldsymbol{C}_{\boldsymbol{\alpha} \boldsymbol{\alpha}}-c_{\alpha_{0} \alpha_{0}}^{-1} \boldsymbol{C}_{\boldsymbol{\alpha} \alpha_{0}} \boldsymbol{C}_{\boldsymbol{\alpha} \alpha_{0}}^{H}
\end{gathered}
$$

where $\boldsymbol{C}_{\boldsymbol{h}}$ is the $L \times L$ time-domain channel covariance matrix, $c_{\alpha_{0} \alpha_{m}}=E\left\{\alpha_{0} \bar{\alpha}_{m}\right\}, 0 \leq l, m \leq N-1$, and $\boldsymbol{C}_{\boldsymbol{\alpha} \alpha_{0}}=\left[c_{\alpha_{1} \alpha_{0}} c_{\alpha_{2} \alpha_{0}} \ldots c_{\alpha_{N-1} \alpha_{0}}\right]^{T}$. Then we can derive the conditional mean and variance of the random variable $z_{k}$ as [17]

$$
\begin{aligned}
E\left\{z_{k} \mid \alpha_{0}, v_{\Delta}\right\} & =\frac{\pi v_{\Delta}}{N}\left|\alpha_{0}\right|^{2} c_{\alpha_{0} \alpha_{0}}^{-1} \Re\left(\boldsymbol{V}_{k}^{T} \boldsymbol{C}_{\boldsymbol{\alpha} \alpha_{0}}\right) \\
& =\frac{\pi v_{\Delta}}{N}\left|\alpha_{0}\right|^{2} a_{k}^{\prime}
\end{aligned}
$$

and

$$
\begin{aligned}
\operatorname{Var}\left(z_{k} \mid \alpha_{0}, v_{\Delta}\right) & =\frac{\pi^{2} v_{\Delta}^{2}}{2 N^{2}}\left|\alpha_{0}\right|^{2} \boldsymbol{V}_{k}^{T} \boldsymbol{C}_{\boldsymbol{\alpha} \mid \alpha_{0}} \overline{\boldsymbol{V}}_{k} \\
& =\frac{\pi^{2} v_{\Delta}{ }^{2}}{2 N^{2}}\left|\alpha_{0}\right|^{2} b_{k}
\end{aligned}
$$

where $a^{\prime}{ }_{k}=c_{\alpha_{0} \alpha_{0}}^{-1} \Re\left(\boldsymbol{V}_{k}^{T} \boldsymbol{C}_{\boldsymbol{\alpha} \alpha_{0}}\right)$ and $b_{k}=\boldsymbol{V}_{k}^{T} \boldsymbol{C}_{\boldsymbol{\alpha} \mid \alpha_{0}} \overline{\boldsymbol{V}}_{k}$. Here $\boldsymbol{P}_{k}$ and $\boldsymbol{V}_{k}$ are related by $\boldsymbol{P}_{k}=\frac{\pi v_{\Delta}}{N} \boldsymbol{V}_{k}$. Rearranging the random variables inside the $Q$ function in (18) yields [17]

$$
\begin{aligned}
P_{b}\left(\xi \mid \alpha_{0}, \boldsymbol{\alpha}, v_{\Delta}\right)=\frac{1}{2^{N-1}} \sum_{k=1}^{2^{N-2}}\{ & Q\left(\mu_{+k}+\lambda_{k} Y_{k}\right) \\
& \left.+Q\left(\mu_{-k}-\lambda_{k} Y_{k}\right)\right\}
\end{aligned}
$$

where $Y_{k} \sim \mathcal{N}(0,1), \mu_{+k}=\frac{\left|\alpha_{0}\right|}{\sigma}\left(1+\frac{\pi}{N} v_{\Delta} a_{k}^{\prime}\right), \mu_{-k}=\frac{\left|\alpha_{0}\right|}{\sigma}(1-$ $\left.\frac{\pi}{N} v_{\Delta} a_{k}^{\prime}\right)$ and $\lambda_{k}=\sqrt{\frac{b_{k}}{2 \sigma^{2}}} \frac{\pi v_{\Delta}}{N}$. Then the BER conditioned on $\alpha$ and $v_{\Delta}$ can be obtained as [23, eq. 3.66]

$$
\begin{aligned}
P_{b}\left(\xi \mid \alpha_{0}, v_{\Delta}\right)=\frac{1}{2^{N-1}} \sum_{k=1}^{2^{N-2}}\{Q & \left(\frac{\mu_{+k}}{\sqrt{1+\lambda_{k}^{2}}}\right) \\
& \left.+Q\left(\frac{\mu_{-k}}{\sqrt{1+\lambda_{k}^{2}}}\right)\right\} .
\end{aligned}
$$

Further manipulating and averaging (23) with respect to $\alpha_{0}$ give

$$
\begin{array}{r}
P_{b}\left(\xi \mid v_{\Delta}\right)=\frac{1}{2}-\frac{1}{2^{N}} \sum_{k=1}^{2^{N-2}}\left\{\frac{M\left(1+m_{k} v_{\Delta}\right)}{2 \sqrt{p+q_{k} v_{\Delta}+r_{k} v_{\Delta}^{2}}}\right. \\
\left.+\frac{M\left(1-m_{k} v_{\Delta}\right)}{2 \sqrt{p-q_{k} v_{\Delta}+r_{k} v_{\Delta}^{2}}}\right\}
\end{array}
$$

where $M=\sqrt{2 \sigma_{R}^{2} \gamma}, \gamma$ is the same as defined before, $m_{k}=\frac{\pi}{N} a^{\prime}{ }_{k}, p=\left(1+2 \sigma_{R}^{2} \gamma\right), q_{k}=\frac{4 \sigma_{R}^{2} \gamma \pi a^{\prime}{ }_{k}}{N}$, and $r_{k}=\frac{\pi^{2}}{N^{2}} \gamma\left(b_{k}+\right.$ $a_{k}^{\prime 2}$ ). Averaging (24) over $v_{\Delta}$, we obtain the BER as

$$
\begin{aligned}
P_{b}(\xi) & =\frac{1}{b 2^{N}} \sum_{k=1, m_{k} \neq 0}^{2^{N-2}}\left[2 b-[A]_{-b}^{b}-[B]_{-b}^{b}\right] \\
& +\frac{1}{b 2^{N}} \sum_{k=1, m_{k}=0}^{2^{N-2}}\left[2 b-\left[\frac{M}{\sqrt{r_{k}}} \sinh ^{-1}\left(\frac{v_{\Delta}}{L^{\prime}}\right)\right]_{-b}^{b}\right]
\end{aligned}
$$

where

$$
\begin{gathered}
A=\frac{M m_{k}}{2 r_{k}}\left[\sqrt{p+q_{k} v_{\Delta}+r_{k} v_{\Delta}^{2}}\right] \\
+\left[\frac{M\left(1-\frac{q_{k} m_{k}}{2 r_{k}}\right)}{2 \sqrt{r_{k}}} \sinh ^{-1}\left(\frac{v_{\Delta}}{L^{\prime}}+\frac{q_{k}}{2 r_{k} L^{\prime}}\right)\right] \\
B=\frac{-M m_{k}}{2 r_{k}}\left[\sqrt{p-q_{k} v_{\Delta}+r_{k} v_{\Delta}^{2}}\right] \\
+\left[\frac{M\left(1-\frac{q_{k} m_{k}}{2 r_{k}}\right)}{2 \sqrt{r_{k}}} \sinh ^{-1}\left(\frac{v_{\Delta}}{L^{\prime}}-\frac{q_{k}}{2 r_{k} L^{\prime}}\right)\right] \\
L^{\prime}=\sqrt{\frac{p}{r_{k}}-\frac{q_{k}^{2}}{4 r_{k}^{2}}}
\end{gathered}
$$

and $L^{\prime}$ is always positive.

\section{AWGN and Frequency-Flat Rayleigh Fading Channels with Perfect Power Control}

To evaluate (7), we should know the pdf of $v_{\Delta}$. As far as ML estimators are concerned, we can observe the nature of the pdf of $v_{\Delta}$ conditioned on the channel. Asymptotic properties of the maximum likelihood estimate (MLE) indicate that if the regularity conditions are satisfied [27], then the MLE of the unknown parameter $\boldsymbol{\theta}$ is asymptotically Gaussian-distributed as

$$
\hat{\boldsymbol{\theta}} \sim \mathcal{N}\left(\boldsymbol{\theta}, \boldsymbol{I}^{-1}(\boldsymbol{\theta})\right)
$$


where $\boldsymbol{I}(\boldsymbol{\theta})$ is the Fisher information matrix evaluated at the true value of the unknown parameter [27]. Hence, it is reasonable to use the conditional pdf of $v_{\Delta}$ as

$$
f\left(v_{\Delta} \mid \boldsymbol{h}\right)=\mathcal{N}\left(0, \boldsymbol{I}^{-1}(\boldsymbol{\theta})\right)=\mathcal{N}\left(0,\left.\mathrm{CRB}\right|_{\boldsymbol{h}}\right)
$$

where $\left.\mathrm{CRB}\right|_{\boldsymbol{h}}$ is the Cramer-Rao lower bound conditioned on the CIR. For a training signal consisting of $(P+1)$ identical parts, each having $L$ samples, and if the CFO estimation is based on $P L$ samples (excluding the cyclic prefix with $L$ samples), the CRB conditioned on the channel $\boldsymbol{h}$ is given by [29]

$$
\left.\mathrm{CRB}\right|_{\boldsymbol{h}}=\frac{3 N^{3} \sigma^{2}}{\pi^{2} P L^{3}\left(P^{2}-1\right) \boldsymbol{h}^{H} \boldsymbol{S}^{H} \boldsymbol{S h}} .
$$

For most of the training designs [29][30], we have $\boldsymbol{S}^{H} \boldsymbol{S}=E_{a v} \boldsymbol{I}$, and hence

$$
\left.\mathrm{CRB}\right|_{\boldsymbol{h}}=\frac{3 N^{3} \sigma^{2}}{\pi^{2} P L^{3}\left(P^{2}-1\right) E_{a v} \boldsymbol{h}^{H} \boldsymbol{h}} .
$$

If we assume perfect power control, we can say $\boldsymbol{h}^{H} \boldsymbol{S}^{H} \boldsymbol{S} \boldsymbol{h}$ is constant and hence for a receiver with a CFO estimator, the pdf of the residual CFO can be considered as a Gaussian pdf independent of the channel resulting simply $f\left(v_{\Delta} \mid \boldsymbol{h}\right)=$ $f\left(v_{\Delta}\right)$. If we consider arbitrary training signal samples $\left\{s_{k}\right\}$, the CRB for $v$ derived for the ML joint estimation of $v$ and $\boldsymbol{h}$ is given by [5]

$$
\left.\mathrm{CRB}\right|_{\boldsymbol{h}}=\frac{N^{2} \sigma^{2}}{4 \pi^{2} \boldsymbol{h}^{H} \boldsymbol{S}^{H} \boldsymbol{\Lambda}\left(\boldsymbol{I}_{N}-\boldsymbol{B}\right) \boldsymbol{\Lambda} \boldsymbol{S h}}
$$

where

$$
\boldsymbol{B}=\boldsymbol{S}\left(\boldsymbol{S}^{H} \boldsymbol{S}\right)^{-1} \boldsymbol{S}^{H}
$$

and $\boldsymbol{\Lambda}=\operatorname{diag}\{0,1, \ldots, N-1\}$. In our derivation, we use (33).

Note that the above ML estimators are derived for a deterministic unknown CFO. However, the pdf of the corresponding residual CFO conditioned on a particular fixed $\mathrm{CFO}$ value is asymptotically Gaussian and is independent of that $\mathrm{CFO}$ value as can be observed from (30). Hence, averaging over the preestimation random $\mathrm{CFO}$ will still give the same pdf of $v_{\Delta}$ as described in (30).

1) AWGN Channel: For the AWGN channel, (7) simply reduces to a single integral evaluation as we do not have to average with respect to the channel. The signal model for AWGN channel can be obtained from (1) as

$$
r=\Gamma(v) s+w
$$

where $s=\left[s_{0} s_{1} \ldots s_{N-1}\right]^{T}$ is the training signal vector. The CRB of the CFO estimation for the signal model in (35) is given by [28]

$$
\mathrm{CRB}=\frac{N^{2} \sigma^{2}}{4 \pi^{2} \boldsymbol{s}^{H} \boldsymbol{\Lambda}^{2} \boldsymbol{s}}=\beta .
$$

Then we evaluate the BER as

$$
P_{b}(\xi)=\frac{1}{2^{N-1}} \sum_{k=1}^{2^{N-2}} 2 E\left\{Q\left(\sqrt{2 \gamma}+\sqrt{2 \gamma} a_{k} v_{\Delta}\right)\right\}
$$

where the expectation is with respect to $v_{\Delta}$. Re-arranging the random variables inside the $Q$ function in (37) gives

$$
\begin{aligned}
P_{b}(\xi) & =\frac{1}{2^{N-1}} \sum_{k=1}^{2^{N-2}} 2 E\left\{Q\left(\sqrt{2 \gamma}+\sqrt{2 \gamma \beta} a_{k} \frac{v_{\Delta}}{\sqrt{\beta}}\right)\right\} \\
& =\frac{1}{2^{N-1}} \sum_{k=1}^{2^{N-2}} 2 E\left\{Q\left(\sqrt{2 \gamma}+\sqrt{2 \gamma \beta} a_{k} X\right)\right\}
\end{aligned}
$$

where $X \sim \mathcal{N}(0,1)$. Then, using [31, eq. 3.66] we obtain the BER as

$$
P_{b}(\xi)=\frac{1}{2^{N-1}} \sum_{k=1}^{2^{N-2}} 2 Q\left(\sqrt{\frac{2 \gamma}{1+2 \gamma \beta a_{k}^{2}}}\right) .
$$

2) Frequency-Flat Rayleigh Fading Channel: When the frequency-flat fading channel is considered, (33) can be reduced to

$$
\left.\mathrm{CRB}\right|_{\alpha}=\frac{2 N^{2}}{\left(8 \pi^{2} \boldsymbol{s}^{H} \boldsymbol{\Lambda}\left(\boldsymbol{I}_{N}-\boldsymbol{B}\right) \boldsymbol{\Lambda} \boldsymbol{s}\right)} \frac{\sigma^{2}}{|\alpha|^{2}}=\frac{\lambda}{|\alpha|^{2}}
$$

where $\alpha$ is complex Gaussian with variance $\sigma_{R}^{2}$ per dimension. Under the perfect power control, we can equivalently consider that $|\alpha|^{2}$ is constant while fixing $s$. Thus we assume the pdf of residual $\mathrm{CFO}$ to be

$$
f\left(v_{\Delta} \mid \alpha\right)=f\left(v_{\Delta}\right)=\mathcal{N}(0, \lambda) .
$$

Using (7), (14), (41) and the same mathematical arguments used in deriving (39), we obtain the BER for the frequencyflat Rayleigh fading channel under perfect power control as

$$
P_{b}(\xi)=\frac{1}{2^{N-1}} \sum_{k=1}^{2^{N-2}} 2 Q\left(\sqrt{\frac{2 \gamma}{1+2 \gamma \lambda a_{k}^{2}}}\right) .
$$

\section{Performance Analysis with Channel-DePEndent Residual CFO}

For the channel-dependent residual CFO scenario, the bit error probability can be expressed as

$$
P_{b}(\xi)=\iint P_{b}\left(\xi \mid v_{\Delta}, \boldsymbol{h}\right) f\left(v_{\Delta} \mid \boldsymbol{h}\right) f(\boldsymbol{h}) d v_{\Delta} d \boldsymbol{h} .
$$

The closed form solution to (43) for the frequency-flat Rayleigh fading channel is presented in the following. However, solving the above problem for the frequency-selective case appears to be intractable and hence we adopt an alternative approach for the frequency-selective case which will be presented in Section V.

\section{A. Frequency-Flat Rayleigh Fading Channel}

The variance of the conditional Gaussian random variable $v_{\Delta} \mid \alpha$ for the frequency-flat Rayleigh fading channel is given by (40) for the MLE estimator [5] we use in this paper. Manipulating the equations (14), (15), (30), (40) and (43) yields the BER conditioned on $\alpha$ as

$$
P_{b}(\xi \mid \alpha)=\frac{1}{2^{N-1}} \sum_{k=1}^{2^{N-2}} 2 Q\left(\frac{\mu}{\sqrt{1+\eta_{k}^{2}}}\right)
$$


where $\mu=\sqrt{2 \gamma}|\alpha|$ and $\eta_{k}=\sqrt{2 \gamma \lambda} a_{k}$. Further averaging with respect to the Rayleigh variable $|\alpha|$, we obtain the BER as

$$
P_{b}(\xi)=\frac{1}{2^{N-1}} \sum_{k=1}^{2^{N-2}}\left\{1-\sqrt{\frac{\frac{2 \gamma}{1+2 \gamma \lambda a_{k}^{2}} \sigma_{R}^{2}}{1+\frac{2 \gamma}{1+2 \gamma \lambda a_{k}^{2}} \sigma_{R}^{2}}}\right\} .
$$

\section{An Alternative ApProach to BER Analysis in FREQUENCY-SELECTIVE CHANNEL}

Since the BER calculation in the frequency-selective fading channel seems to be intractable using the procedure used earlier, we propose the following method with relaxed assumptions and we denote this as an analysis with relaxed assumptions. In this analytical development we assume that $v_{\Delta}$ and $\boldsymbol{h}$ are independent and $v_{\Delta}$ is uniformly distributed. Even though these assumptions are not entirely justifiable for the MLE1 estimator in [5], analytical results so obtained closely match with the simulation results. We applied this approach for both frequency-flat and frequency-selective scenarios as follows.

\section{A. Frequency-Flat Rayleigh Fading Channel}

The estimates of $v$ and $h_{0}$ can be written as [5]

$$
\begin{aligned}
\hat{v} & =\arg _{\tilde{v}} \max \left\{\boldsymbol{r}^{H} \Gamma(\tilde{v}) \boldsymbol{B} \Gamma^{H}(\tilde{v}) \boldsymbol{r}\right\} \\
\hat{h_{0}} & =\left(\boldsymbol{S}^{H} \boldsymbol{S}\right)^{-1} \boldsymbol{S}^{H} \Gamma^{H}(\hat{v}) \boldsymbol{r}
\end{aligned}
$$

where $\boldsymbol{B}$ is given in (34) and $\boldsymbol{S}=\boldsymbol{s}$ since $L=1$. Substituting (1) into (47) and using the approximation $e^{\frac{-j 2 \pi l v \Delta}{N}} \simeq(1-$ $\left.\frac{j 2 \pi l v_{\Delta}}{N}\right)$ for very small $v_{\Delta}$, we can find an approximation for $\hat{h}_{0}$ as

$$
\hat{h_{0}} \approx\left[1-\frac{j 2 \pi v_{\Delta}}{N^{2}} \sum_{k=1}^{N-1} k\left|s_{k}\right|^{2}\right] h_{0}+w_{n e w}
$$

where $w_{n e w}$ is a zero-mean circularly-symmetric complex Gaussian variable with variance $\frac{\sigma^{2}}{N}$ per dimension. For simplicity we define $q=\left[1-\frac{j 2 \pi v_{\Delta}}{N^{2}} \sum_{k=1}^{N-1} k\left|s_{k}\right|^{2}\right]$. From (13), we have

$$
R_{0}=\left[1+\frac{\pi v_{\Delta}}{N} \sum_{l=1}^{N-1} c_{l} a_{l}\right] h_{0}+n_{0}^{\prime} ; l=0,1, \cdots, N-1
$$

where we have used $I_{l}^{\prime}=\frac{\pi v_{\Delta}}{N} a_{l}$ for $l \neq 0$ and $I_{0}^{\prime}=1$ under very small $v_{\Delta}$ assumption, and $c_{0}=1$. Let $p=\left[1+\frac{\pi v_{\Delta}}{N} \sum_{l=1}^{N-1} c_{l} a_{l}\right]$. Now we want to find $\operatorname{Pr}\left[\Re\left(\frac{R_{0}}{\hat{h}_{0}}\right)<0 \mid c_{0}=1\right]$. Applying the results from Appendix B of [33], we obtain

$$
P_{b}\left(\xi \mid v_{\Delta}, c_{1}, c_{2}, \ldots c_{N-1}\right)=1-\frac{v_{2}}{v_{1}+v_{2}}
$$

where

$$
\begin{gathered}
v_{1}=\sqrt{w^{2}+\frac{1}{4\left(\mu_{\hat{h_{0}} \hat{h_{0}}} \mu_{R_{0} R_{0}}-\left|\mu_{\hat{h_{0} R_{0}}}\right|^{2}\right)}}-w \\
v_{2}=\sqrt{w^{2}+\frac{1}{4\left(\mu_{\hat{h_{0}} \hat{h_{0}}} \mu_{R_{0} R_{0}}-\left|\mu_{\hat{h_{0} R_{0}}}\right|^{2}\right)}}+w \\
w=\frac{\mu_{\hat{h_{0} R_{0}}}+\mu_{\hat{h_{0} R_{0}}}^{*}}{4\left(\mu_{\hat{\hat{0}_{0}} \hat{h_{0}}} \mu_{R_{0} R_{0}}-\left|\mu_{\hat{h_{0} R_{0}}}\right|^{2}\right)}
\end{gathered}
$$

and $\mu_{X Y}=\frac{1}{2} E\left[(X-E\{X\})(Y-E\{Y\})^{*}\right]$. Furthermore, we can derive the following statistical relationships conditioned on $v_{\Delta}$ and all data symbols $\left(\begin{array}{lllll}c_{1} & c_{2} & \cdots & c_{N-1}\end{array}\right)$ : $\mu_{R_{0} R_{0}}=\frac{|p|^{2} \sigma_{h_{0}}^{2}}{2}+\sigma^{2}, \mu_{\hat{h_{0}} \hat{h_{0}}}=\frac{|q|^{2} \sigma_{h_{0}}^{2}}{2}+\frac{\sigma^{2}}{N}, \mu_{\hat{h_{0} R_{0}}}=$ $\frac{p^{*} q \sigma_{h_{0}}^{2}}{2}, \mu_{R_{0} \hat{h_{0}}}=\frac{q^{*} p \sigma_{h_{0}}^{2}}{2}, w=\frac{\Re\left(p q^{*}\right)}{2 \sigma^{2}\left(\frac{|p|^{2}}{N}+|q|^{2}+\frac{2 \sigma^{2}}{N \sigma_{h_{0}}^{2}}\right)}$ where $\sigma_{h_{0}}^{2}$ is the variance of $h_{0}$. After some algebraic manipulations, we can obtain the conditional probability (54), where $a_{1}^{\prime}=0.5, b_{1}^{\prime}=-\frac{\pi a}{2 N}, c_{1}^{\prime}=-\frac{\pi^{2} g \lambda}{N^{3}}, a_{2}^{\prime}=\frac{2 \sigma^{2}}{\sigma_{h_{0}}^{2}}\left(1+\frac{1}{N}+\right.$ $\left.\frac{2 \sigma^{2}}{N \sigma_{h_{0}}^{2}}\right), b_{2}^{\prime}=-\frac{2 \sigma^{2}}{\sigma_{h_{0}}^{2}}\left(\frac{2 \pi a}{N^{2}}\right), c_{2}^{\prime}=\frac{2 \sigma^{2}}{\sigma_{h_{0}}^{2}}\left[\frac{\pi^{2}}{N^{3}}\left(a^{2}+g^{2}\right)+\frac{4 \pi^{2} \lambda^{2}}{N^{4}}\right]$, $a_{3}^{\prime}=\left(4 a_{1}^{\prime 2}+a_{2}^{\prime}\right), b_{3}^{\prime}=\left(8 a_{1}^{\prime} b_{1}^{\prime}+b_{2}^{\prime}\right), c_{3}^{\prime}=\left[4\left(b_{1}^{2}+2 a_{1}^{\prime} c_{1}^{\prime}\right)+c_{2}^{\prime}\right]$, $d_{3}^{\prime}=8 b_{1}^{\prime} c_{1}^{\prime}, e_{3}^{\prime}=4 c_{1}^{2}, a=\sum_{l=1}^{N-1} c_{l} \cot \left(\frac{\pi l}{N}\right), \lambda=\sum_{k=1}^{N-1} k\left|s_{k}\right|^{2}$ and $g=\sum_{l=1}^{N-1} c_{l}$. Averaging (54) over all possible data symbol combinations and $v_{\Delta}$ yields BER as in (55).

In general, closed form solutions do not exist for (55). But a closed form solution can be derived ignoring the terms with coefficients $d_{3}{ }^{\prime}$ and $e_{3}{ }^{\prime}$. This is really the case when $v_{\Delta} \rightarrow 0$. However, at high SNR this is not acceptable and we have to use numerical integration techniques given in software packages such as MatLab or Mathematica.

\section{B. Frequency-Selective Rayleigh Fading Channel}

The estimate of the channel coefficient vector is obtained as [5]

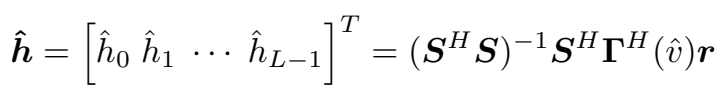

where $\boldsymbol{S}, \boldsymbol{\Gamma}, \boldsymbol{r}$ are as defined in (1) and $\hat{v}$ is the estimate of the normalized CFO. Denoting $\hat{\boldsymbol{\alpha}}_{e}$ as the $N$-point DFT of $\hat{\boldsymbol{h}}$, i.e., $\hat{\boldsymbol{\alpha}}_{e}=\left[\begin{array}{llll}\hat{\alpha}_{0} & \hat{\alpha}_{1} & \ldots & \hat{\alpha}_{N-1}\end{array}\right]^{T}=\sqrt{N} \boldsymbol{F}_{L} \hat{\boldsymbol{h}}$, and after some matrix manipulations, we obtain

$$
\hat{\alpha}_{0}=\frac{1}{\sqrt{N}} \mathbf{1}_{N}^{T} \boldsymbol{A}_{e} \boldsymbol{F}^{H}\left[\alpha_{0} \boldsymbol{\alpha}^{T}\right]^{T}+\mathbf{1}_{N}^{T} \boldsymbol{B}_{e} \boldsymbol{w}
$$

$$
\begin{gathered}
P_{b}\left(\xi \mid v_{\Delta}, c_{1}, c_{2}, \ldots c_{N-1}\right)=\frac{1}{2}-\frac{a_{1}^{\prime}+b_{1}^{\prime} v_{\Delta}+c_{1}^{\prime} v_{\Delta}{ }^{2}}{\sqrt{a_{3}^{\prime}+b_{3}^{\prime} v_{\Delta}+c_{3}^{\prime} v_{\Delta}{ }^{2}+d_{3}^{\prime} v_{\Delta}{ }^{3}+e_{3}^{\prime} v_{\Delta}{ }^{4}}} \\
P_{b}(\xi)=\frac{1}{2^{N-1}} \sum_{c_{1} \in\{-1,1\}} \sum_{c_{2} \in\{-1,1\}} \ldots \ldots \sum_{c_{N-1} \in\{-1,1\}} \frac{1}{2 b} \times\left[\int_{-b}^{b} \frac{1}{2}-\frac{a_{1}^{\prime}+b_{1}{ }^{\prime} v_{\Delta}+c_{1}^{\prime} v_{\Delta}{ }^{2}}{\sqrt{a_{3}^{\prime}+b_{3}{ }^{\prime} v_{\Delta}+c_{3}{ }^{\prime} v_{\Delta}{ }^{2}+d_{3}{ }^{\prime} v_{\Delta}{ }^{3}+e_{3}{ }^{\prime} v_{\Delta}{ }^{4}}} d v_{\Delta}\right]
\end{gathered}
$$


where

$$
\begin{aligned}
\boldsymbol{A}_{e} & =\left(\begin{array}{cc}
\boldsymbol{A} & \mathbf{0}_{L \times(N-L)} \\
\mathbf{0}_{(N-L) \times L} & \mathbf{0}_{(N-L) \times(N-L)}
\end{array}\right) \\
\boldsymbol{B}_{\boldsymbol{e}} & =\left[\left(\left(\boldsymbol{S}^{H} \boldsymbol{S}\right)^{-1} \boldsymbol{S}^{H}\right)^{T}, \mathbf{0}_{N \times(N-L)}\right]^{T} \\
\boldsymbol{A} & =\left(\boldsymbol{S}^{H} \boldsymbol{S}\right)^{-1} \boldsymbol{S}^{H} \boldsymbol{\Gamma}^{H}\left(v_{\Delta}\right) \boldsymbol{S} .
\end{aligned}
$$

From (14) when $c_{0}=1$, we can deduce that

$$
R_{0}=\alpha_{0}+a \boldsymbol{P} \boldsymbol{\alpha}+n_{0}^{\prime}
$$

where $a=\frac{\pi v_{\Delta}}{N}, \quad P=\left[c_{1} a_{1} c_{2} a_{2} \cdots c_{N-1} a_{N-1}\right]^{T}$, and we have used (6) with $I_{l}^{\prime}=a a_{l}$ for $l \neq 0$ and $I_{0}^{\prime}=1$ under very small $v_{\Delta}$ assumption. Here we apply the same procedure which was used to derive (55). The random variables $\hat{\alpha}_{0}$ and $R_{0}$ conditioned on $v_{\Delta}$ and the data sequence $\left[\begin{array}{cccc}c_{1} & c_{2} & \cdots & c_{N-1}\end{array}\right]^{T}$ are complex Gaussian. Hence we can derive the following statistics:

$$
\mu_{R_{0} R_{0}}=A_{1}+B_{1} v_{\Delta}+C_{1} v_{\Delta}^{2}
$$

where $\quad A_{1}=\frac{1}{2}\left[2 \sigma^{2}+c_{\alpha_{0} \alpha_{0}}\right], \quad B_{1}=\frac{\pi}{N} \Re\left(\boldsymbol{C}_{\boldsymbol{\alpha} \alpha_{0}}^{H} \overline{\boldsymbol{P}}\right)$, $C_{1}=\frac{\pi^{2}}{2 N^{2}}\left[\boldsymbol{P}^{T} \boldsymbol{C}_{\boldsymbol{\alpha} \alpha} \overline{\boldsymbol{P}}\right]$, and

$$
\mu_{\hat{\alpha_{0}} \hat{\alpha_{0}}}=\frac{1}{2}\left[\mathbf{1}_{N}^{T} \boldsymbol{A}_{e} \boldsymbol{C}_{h} \boldsymbol{A}_{e}^{H} \mathbf{1}_{\boldsymbol{N}}+2 \sigma^{2} \mathbf{1}_{N}^{T} \boldsymbol{B}_{e} \boldsymbol{B}_{e}^{H} \mathbf{1}_{\boldsymbol{N}}\right] .
$$

With the assumption of independent and identically distributed (iid) time-domain channel coefficients and using the relation $e^{\frac{-j 2 \pi l v_{\Delta}}{N}} \approx\left(1-\frac{j 2 \pi l v_{\Delta}}{N}\right)$ for very small $v_{\Delta}$ values, we can deduce from (60) that

$$
\mu_{\hat{\alpha_{0}} \hat{\alpha_{0}}}=\frac{1}{2}+\frac{r}{2}+\frac{1}{2 L} \mu v_{\Delta}^{2}
$$

where $\quad r=2 \sigma^{2} \mathbf{1}_{N}^{T} \boldsymbol{B}_{e} \boldsymbol{B}_{e}^{H} \mathbf{1}_{\boldsymbol{N}}, \quad \mu=\sum_{i=0}^{L-1}\left|\sum_{m=0}^{L-1} q_{m i}\right|^{2}$, $q_{m i}=\frac{2 \pi j}{N} \sum_{k=0}^{N-1} k\left[\left(\boldsymbol{S}^{H} \boldsymbol{S}\right)^{-1} \boldsymbol{S}^{H}\right]_{m, k}[\boldsymbol{S}]_{k, i}$. $\quad$ Let us denote $\quad A_{2}=\frac{1}{2}, \quad B_{2}=\frac{r}{2}, \quad C_{2}=\frac{1}{2 L} \mu, \quad \boldsymbol{D}=\left[c_{\alpha_{0} \alpha_{0}} \boldsymbol{C}_{\boldsymbol{\alpha} \alpha_{0}}^{H}\right]$, and $\boldsymbol{E}=\boldsymbol{P}^{T}\left[\boldsymbol{C}_{\boldsymbol{\alpha} \alpha_{0}} \boldsymbol{C}_{\boldsymbol{\alpha} \boldsymbol{\alpha}}\right]$ for the notational simplicity. Then

$$
\mu_{R_{0} \hat{\alpha_{0}}}=A_{3}+B_{3} v_{\Delta}+C_{3} v_{\Delta}^{2}
$$

where $\quad A_{3}=\frac{\lambda_{2}}{2 \sqrt{N}}, \quad B_{3}=\left[\frac{\mu_{2}}{2 \sqrt{N}}+\frac{\pi \lambda_{3}}{2 \sqrt{N} N}\right], \quad C_{3}=\frac{\pi \mu_{3}}{2 \sqrt{N} N}$, $\lambda_{2}=\sum_{m=0}^{L-1}[\boldsymbol{D F}]_{1, m}, \quad \mu_{2}=\sum_{m=0}^{L-1}\left([\boldsymbol{D F}]_{1, m} b_{m}^{*}\right)$, $\lambda_{3}=\sum_{m=0}^{L-1}[\boldsymbol{E F}]_{1, m}, \quad \mu_{3}=\sum_{m=0}^{L-1}\left([\boldsymbol{E} \boldsymbol{F}]_{1, m} b_{m}^{*}\right) \quad$ and $b_{m}=\sum_{k=0}^{L-1} q_{k m}$. Further, we can derive the corresponding $w$ in (53) as

$$
w=\frac{\mu_{\hat{\alpha_{0}} R_{0}}+\mu_{\hat{\alpha}_{0} R_{0}}^{*}}{4\left(\mu_{\hat{\alpha_{0}} \hat{\alpha_{0}}} \mu_{R_{0} R_{0}}-\left|\mu_{\hat{\alpha_{0}} R_{0}}\right|^{2}\right)}=\frac{K}{M}
$$

where $K=\left(g_{1}+g_{2} v_{\Delta}+g_{3} v_{\Delta}^{2}\right), \quad M=4\left[\left(A_{1} A_{2}+A_{1} B_{2}-\right.\right.$ $\left.\left|A_{3}\right|^{2}\right)+\left(B_{1} A_{2}+B_{1} B_{2}-2 \Re\left(A_{3} B_{3}^{*}\right)\right) v_{\Delta}+\left(A_{1} C_{2}+C_{1} B_{2}+\right.$ $\left.C_{1} A_{2}-\left|B_{3}\right|^{2}-2 \Re\left(A_{3} C_{3}^{*}\right)\right) v_{\Delta}^{2}+\left(B_{1} C_{2}-2 \Re\left(B_{3} C_{3}\right)\right) v_{\Delta}^{3}+$ $\left.\left(C_{1} C_{2}-\left|C_{3}\right|^{2}\right) v_{\Delta}^{4}\right], \quad g_{1}=2 \Re\left(A_{3}\right), \quad g_{2}=2 \Re\left(B_{3}\right) \quad$ and $g_{3}=2 \Re\left(C_{3}\right)$. After some algebraic manipulations and using (60)-(63), we can come up with the following conditional error probability (64), [33], where $g_{4}=g_{1}^{2}+4\left(A_{1} A_{2}+A_{1} B_{2}-\left|A_{3}\right|^{2}\right)$, $g_{5}=2 g_{1} g_{2}+4\left(B_{1} A_{2}+B_{1} B_{2}-2 \Re\left(A_{3} B_{3}^{*}\right)\right)$, $g_{6}=\left(g_{2}^{2}+2 g_{1} g_{3}\right)+4\left(A_{1} C_{2}+C_{1} B_{2}+C_{1} A_{2}-\left|B_{3}\right|^{2}-\right.$ $\left.2 \Re\left(A_{3} C_{3}^{*}\right)\right), \quad g_{7}=2 g_{2} g_{3}+4\left(B_{1} C_{2}-2 \Re\left(B_{3} C_{3}\right)\right), \quad$ and $g_{8}=g_{3}^{2}+4\left(C_{1} C_{2}-\left|C_{3}\right|^{2}\right)$. Averaging (64) over all possible data symbol combinations and $v_{\Delta}$ yields BER which is given in (65).

We have to use numerical integration techniques as no closed form solutions are available to evaluate (65).

\section{Simulation Results AND Discussion}

\section{A. Channel-Independent CFO (Uniformly Distributed CFO)}

We consider an OFDM system with $N=64$ and BPSK modulation. The normalized CFO is uniformly distributed over $[-b, b]$ with $b=0.05$ and $b=0.1$.

Fig. 1 shows the BER performance in the AWGN channel. The performance for the ideal situation with no CFO is also included as a reference in Fig. 1. The simulation results for $b=0.05$ case match well with those calculated in (12) but there is a slight discrepancy for $b=0.1$ case especially at low BER values (say below $10^{-3}$ ). This discrepancy is simply due to the fact that the small CFO assumption in the analytical development is not closely matched by the uniform CFO with $b=0.1$, and at these low BER values the CFO has a more dominant effect on BER than the noise does. Further, we can see the deviation of the BER curves for deterministic fixed normalized CFO values of 0.1 and 0.05 , from their counterparts of random CFO with $b=0.1$ and $b=0.05$ respectively. Hence, we can say that considering $\mathrm{CFO}$ as a constant, would not give accurate BER values for practical OFDM systems, as in general CFO is random.

Fig. 2 presents how the BER varies with $b$. For $b<0.1$ (typically acceptable CFO variations), analytical results closely agree with the true values (simulated points), and thus we can say that as long as the (residual) CFO is considerably small, our analytical expressions yield highly accurate results.

The results for frequency-flat and frequency-selective Rayleigh fading channels are presented in Fig. 3 and 4, respectively. The simulation results agree well with our analytical results for both $b=0.05$ and $b=0.1$ cases in both channels, confirming the accuracy of our analytical expressions. We can also see how the BER curves for the constant CFO values deviate from their counterparts for the random CFO. It is interesting to observe that the BER difference at high SNR between $b=0.05$ and $b=0.1$ is indistinguishable for the frequency flat fading (Fig. 3) but noticeable for the frequency selective fading (Fig. 4). This can be explained as follows.

$$
\begin{gathered}
P_{b}\left(\xi \mid v_{\Delta}, c_{1}, c_{2}, \ldots c_{N-1}\right)=\frac{1}{2}-\frac{g_{1}+g_{2} v_{\Delta}+g_{3} v_{\Delta}{ }^{2}}{2 \sqrt{g_{4}+g_{5} v_{\Delta}+g_{6} v_{\Delta}^{2}+g_{7} v_{\Delta}{ }^{3}+g_{8} v_{\Delta}^{4}}} \\
P_{b}(\xi)=\frac{1}{2^{N-1}} \sum_{c_{1} \in\{-1,1\}} \sum_{c_{2} \in\{-1,1\}} \ldots \ldots \sum_{c_{N-1} \in\{-1,1\}} \frac{1}{2 b} \times\left[\int_{-b}^{b} \frac{1}{2}-\frac{g_{1}+g_{2} v_{\Delta}+g_{3} v_{\Delta}{ }^{2}}{2 \sqrt{g_{4}+g_{5} v_{\Delta}+g_{6} v_{\Delta}{ }^{2}+g_{7} v_{\Delta}{ }^{3}+g_{8} v_{\Delta}}} d v_{\Delta}\right]
\end{gathered}
$$




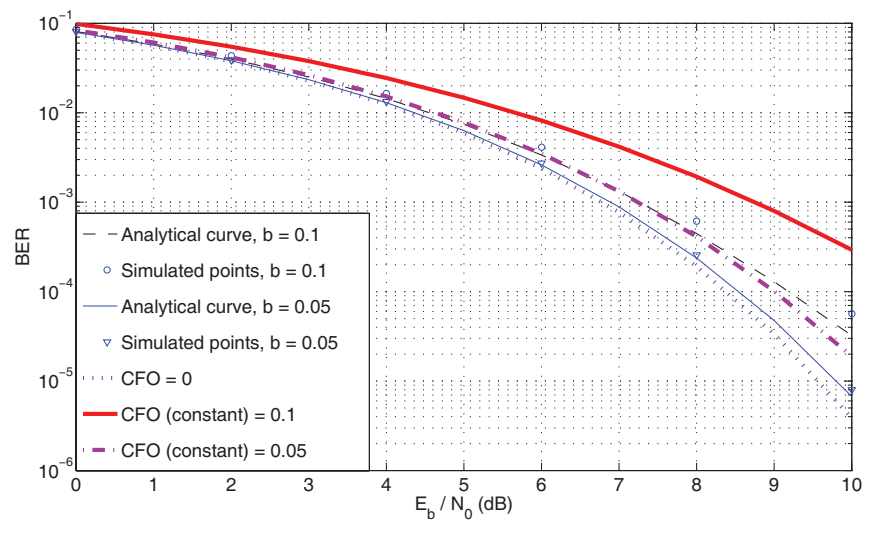

Fig. 1. BER curves for AWGN channel with $N=64$ subcarriers and $b=0.1$, $b=0.05$.

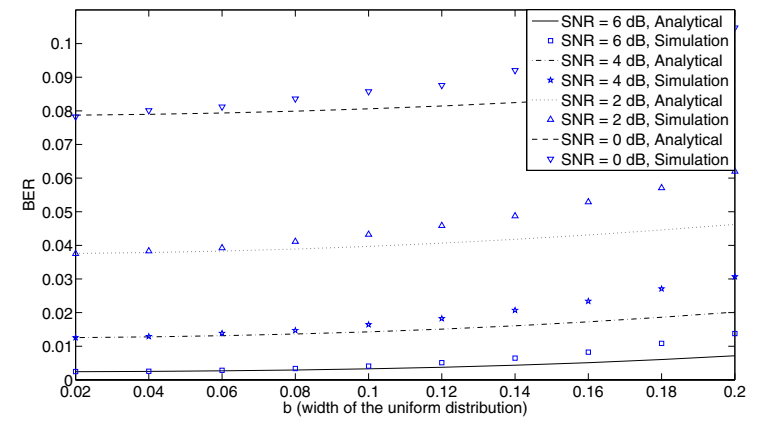

Fig. 2. The variation of BER versus $b$ for $N=64$ subcarriers.

The frequency selective fading causes ICI power fluctuation across the subcarriers signifying unequal error probabilities of different subcarriers in each channel realization, while this is not the case in the frequency flat channel. A larger CFO amplifies this ICI power imbalance in the frequency selective fading channel resulting in a larger CFO-sensitivity as well as a more obvious error floor for the frequency selective channel.

\section{B. Channel-Independent Residual CFO (Perfect Power Con- trol)}

In our analytical development related to this section, the residual CFO is modeled as a Gaussian random variable. Simulation in this section is performed under two settings:

1) Setting $\mathbf{I}$ : The residual CFO is directly generated from a Gaussian density with the variance determined by the CRB conditioned on the channel. A CFO estimator is not used. The purpose of this setting is to verify the theoretical derivation.

2) Setting II : We apply CFO estimation and compensation at the receiver to show the accuracy of our analytical results for practical systems. For the frequency-flat fading channel, we use the CFO estimator (MLE1) from [5]. For the AWGN channel, we can derive the ML CFO estimator based on the signal model in (35) as

$$
\hat{v}=\arg _{\tilde{v}} \max \Re\left\{\sum_{n=0}^{N-1} r[n] s^{*}[n] \exp \left(\frac{-j 2 \pi \tilde{v} n}{N}\right)\right\} .
$$

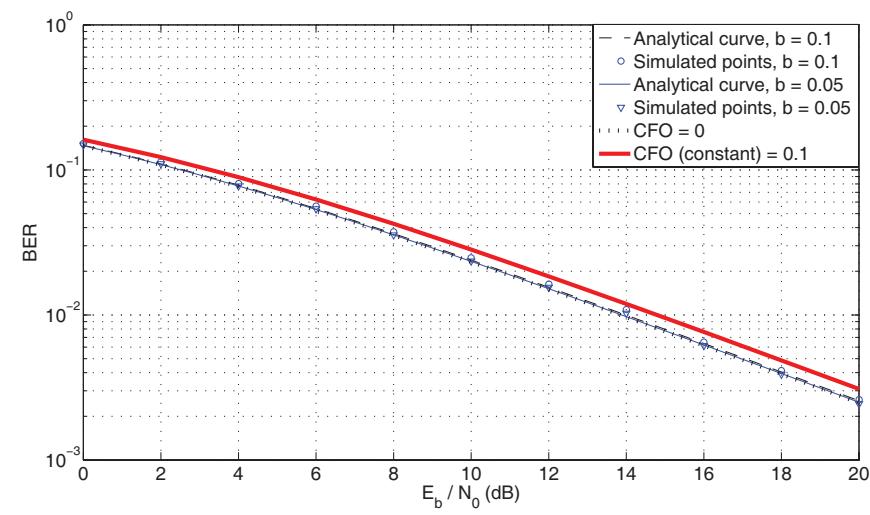

Fig. 3. BER curves for the frequency-flat Rayleigh fading channel with $N=64$ subcarriers and $b=0.1, b=0.05$.

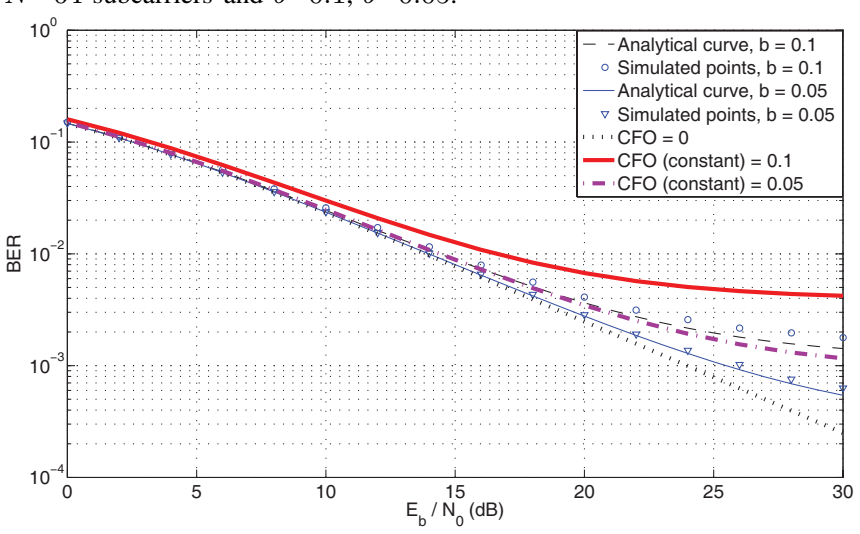

Fig. 4. BER curves for the frequency-selective Rayleigh fading channel with $N=64$ subcarriers, $L=5$ CIR tap coefficients and $b=0.1, b=0.05$.

We use an OFDM system with $N=64$ in a quasistatic channel. In our simulation we have one OFDM preamble/training symbol followed by only one OFDM data symbol. In our analytical derivation we did not consider the CFO-induced, symbol-index-dependent phase shift of $\exp \left(j 2 \pi v \Delta m\left(N+N_{g}\right) / N\right)$ where $m$ is the OFDM symbol index and $N_{g}$ is the number of guard samples. We simply assume that every symbol is phase synchronized so that we can neglect the above phase shift.

For the AWGN channel, the simulation and analytical results for the Setting I and II are presented in Fig. 5 and 6 , respectively. We observe an excellent match between the analytical and simulation results in both figures which confirm the accuracy of our derivation and the applicability of our results to practical systems with a CFO estimator. The results for the frequency-flat Rayleigh fading channel are shown in Fig. 7 and 8 for the Setting I and II, respectively. A marginal mismatch between the simulation and the analytical results is observed for both settings at low SNR values. This slight mismatch can be ascribed to the fact that the small $v_{\Delta}$ approximation used in the analytical derivation is not justified by occasional large CFO estimation errors (outliers) which occur more often at lower SNR values in the simulation. Note that in practice if the channel is in deep fade the receiver will not be able to detect the signal. Hence, the above marginal mismatch is not a concern for practical systems. Also note that we can easily apply our analytical derivation to periodic 


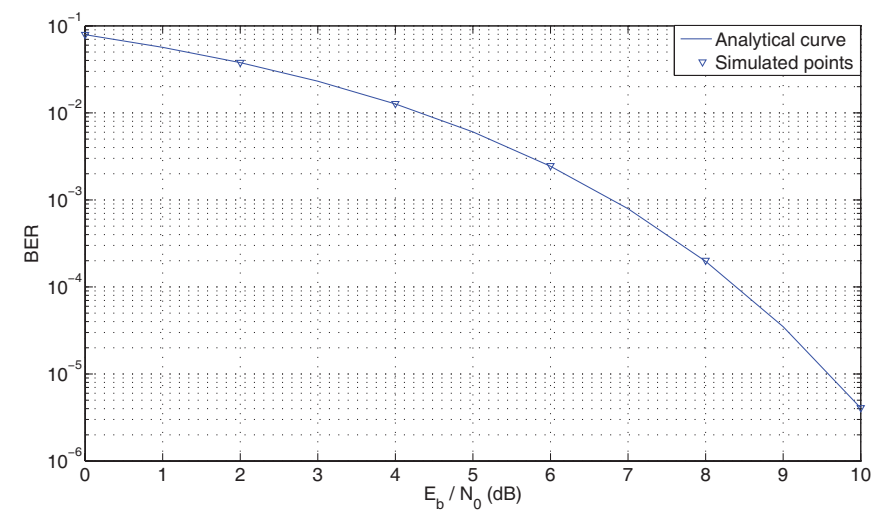

Fig. 5. BER curves for the AWGN channel (setting I) with $N=64$ subcarriers.

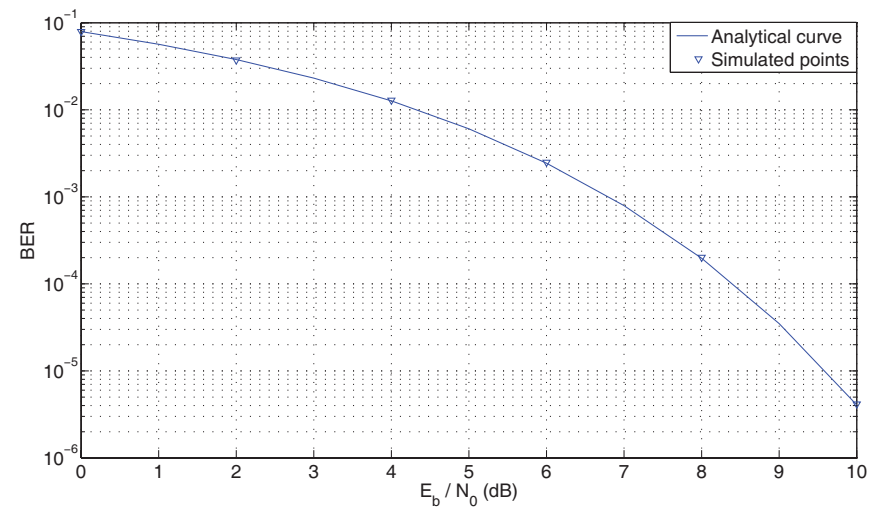

Fig. 6. BER curves for the AWGN channel (setting II) with $N=64$ subcarriers.

training signals by using the CRB in (31).

\section{Channel-Dependent Residual CFO (No Power Control)}

In our analytical derivation corresponding to this section, the residual CFO is modeled as a Gaussian random variable conditioned on the channel realization. Here we use the same simulation settings as described in the previous section. The results for the frequency-flat Rayleigh fading channel are shown in Fig. 9 and 10 for the Setting I and II, respectively. Simulation results closely match the analytical curves verifying the accuracy and practical applicability of our BER analysis.

\section{Channel-Dependent Residual CFO (With Relaxed Assump- tions)}

In the simulation, we apply the MLE1 estimator from [5] and hence the residual CFO is channel-dependent. However, in the analytical derivation related to this section, we assume that the residual $\mathrm{CFO}$ is uniformly distributed over the range $[-b, b]$ and is independent of the channel. We set the variance of the uniform residual CFO of the analytical derivation to be the same as the mean-square error (MSE) of the practical estimator in the simulation which gives the relation $b=\sqrt{3 \mathrm{MSE}}$. Note that the MSE of the CFO estimator depends on the SNR $\left(E_{b} / N_{0}\right)$ and hence we set $b$ according to the MSE at the SNR we are evaluating.

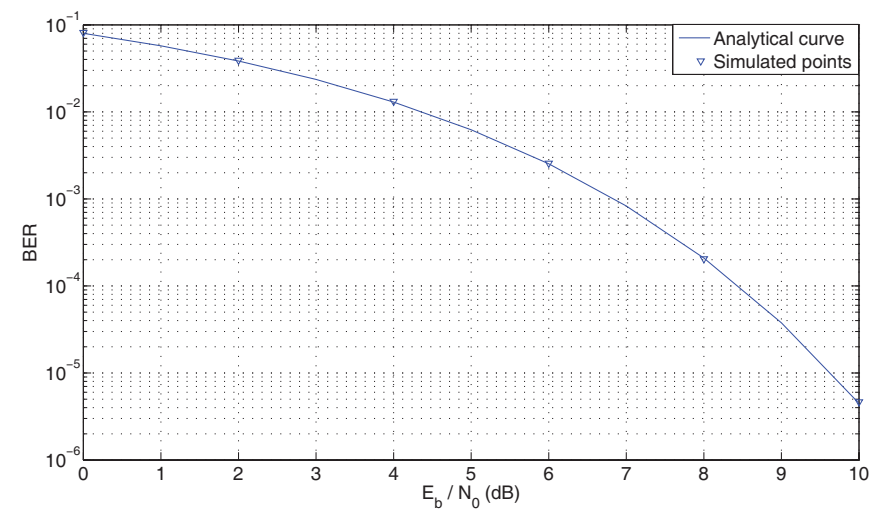

Fig. 7. BER curves for the frequency-flat Rayleigh fading channel (setting I) with perfect power control and $N=64$ subcarriers.

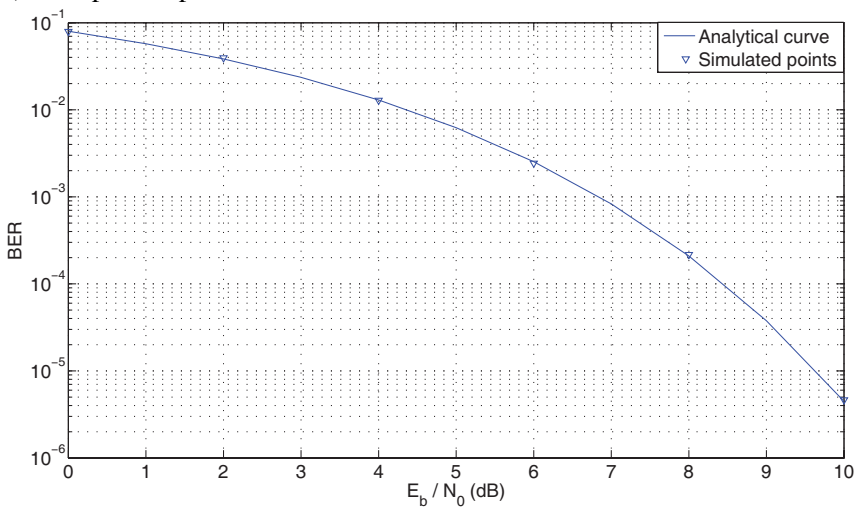

Fig. 8. BER curves for the frequency-flat Rayleigh fading channel (setting II) with perfect power control and $N=64$ subcarriers.

Fig. 11 shows the analytical BER results and the simulation results obtained with the $\mathrm{CFO}$ and channel estimation in frequency-flat and frequency-selective Rayleigh fading channels for OFDM systems with $N=64$. The results show a close match between the simulated and analytical results even with the relaxed assumptions we made in the derivations of (55) and (65). In particular, the analytical results based on the relaxed assumption for the frequency-selective fading channel is quite appealing since the exact BER analysis appears to be intractable.

\section{CONCLUSION}

The CFO or residual CFO, a random variable by nature, is unavoidable in practical OFDM systems and can affect the BER performance. Different from the existing works in the open literature which treated the $\mathrm{CFO}$ as a deterministic variable or which used the Gaussian ICI approximation in the BER analysis, in this paper we have presented BER expressions for BPSK OFDM systems with random (residual) CFO. Using a novel approximation of (residual) CFO-induced ICI coefficients, we have derived closed form BER expressions of OFDM systems over AWGN, frequency-flat and frequencyselective Rayleigh fading channels for both cases of channel independent and channel-dependent random (residual) CFO. Simulation results verify the accuracy of our analytical results and their applicability to practical OFDM systems. 


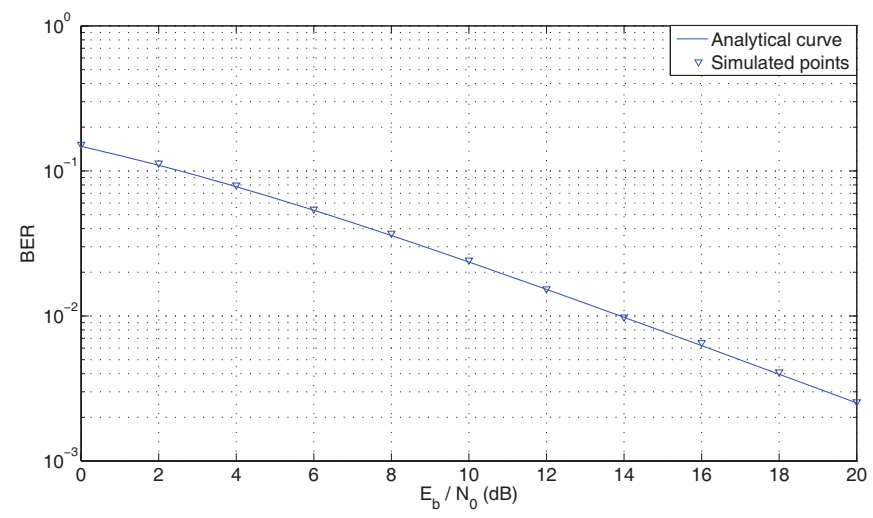

Fig. 9. BER curves for the frequency-flat Rayleigh fading channel (setting I) with no power control and $N=64$ subcarriers.

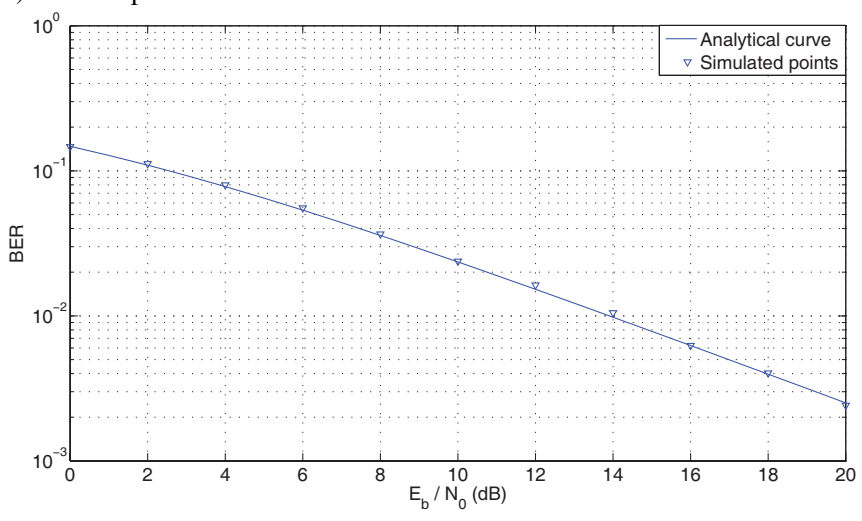

Fig. 10. BER curves for the frequency-flat Rayleigh fading channel (setting II) with no power control and $N=64$ subcarriers.

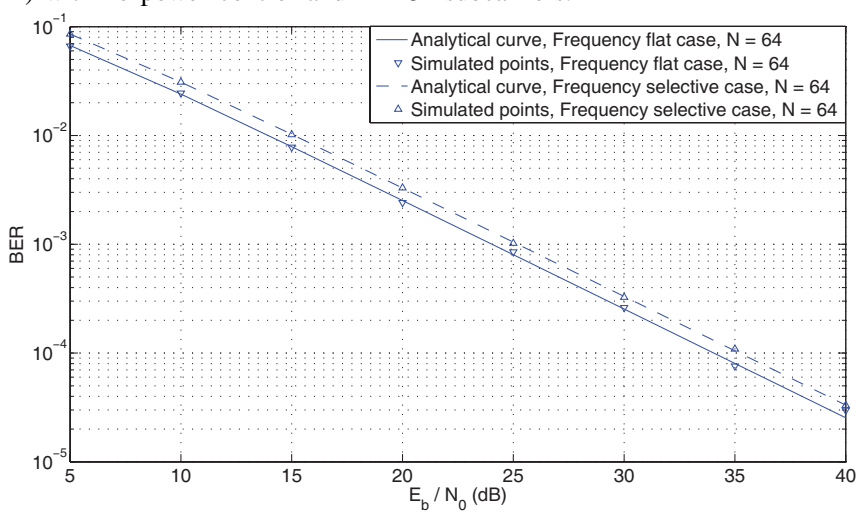

Fig. 11. BER curves for the frequency-flat Rayleigh fading channel $(N=64)$ and the frequency-selective Rayleigh fading channel $(N=64)$; (with relaxed assumptions for the analytical curves)

\section{ACKNOWLEDGMENT}

The authors would like to thank the anonymous reviewers for their comments which have improved the presentation and the quality of the paper.

\section{REFERENCES}

[1] B. Stantchev and G. Fettweis, "Time-variant distortions in OFDM," IEEE Commun. Lett., vol. 4, pp. 312-314, Sept. 2000.

[2] P. H. Moose, "A Technique for orthogonal frequency division multiplexing frequency offset correction," IEEE Trans. Commun., vol. 42, no.10, pp. 2908-2914, Oct. 1994.

[3] T. M. Schmidl and D. C. Cox, "Robust frequency and timing synchronisation for OFDM," IEEE Trans. Commun., vol. 45, no. 12, pp. 16131621, Dec. 1997.
[4] M. Morelli and U. Mengali,"An improved frequency offset estimator for OFDM applications," IEEE Commun. Lett., vol. 3, no. 3, pp. 75-77, Mar. 1999.

[5] M Morelli and U Mengali, "Carrier-frequency estimation for transmission over selective channels," in IEEE Trans. Commun., vol. 48, pp. 1580-1589, Sept. 2000.

[6] J. Lei and T-S. Ng, "A consistent OFDM carrier frequency offset estimator based on distinctively spaced pilot tones," IEEE Trans. Wireless Commun., vol. 3 no. 2, pp. 588-599, Mar. 2004.

[7] Y. Li, H. Minn, N. Al-Dhahir, and R. Calderbank, "Pilot Designs for Consistent Frequency-Offset Estimation in OFDM Systems," IEEE Trans. Commun., vol. 55, no. 5, pp. 864-877, May 2007.

[8] J-J Van de Beek, M. Sandell and P. O. Börjesson , "ML estimation of time and frequency offset in OFDM systems," IEEE Trans. Signal Proc., vol. 45, no. 7, pp. 1800-1805, July 1997.

[9] U. Tureli, H. Liu, and M. D. Zoltowski, "OFDM blind carrier offset estimation:ESPRIT," IEEE Trans. Commun.,vol. 48, pp. 1459-1461, Sept. 2000.

[10] Y. Zhao and S. G. Häggman, "Sensitivity to Doppler shift and carrier frequency errors in OFDM systems-the consequences and solutions," IEEE VTC'96,vol. 3, pp. 1564-1568, Apr.-May 1996.

[11] J. Armstrong, "Analysis of new and existing methods of reducing intercarrier interference due to carrier frequency offset in OFDM," IEEE Trans. Commun., vol. 47, pp. 365-369, Mar. 1999.

[12] M. Russell and G. L. Stüber, "Interchannel interference analysis for OFDM in a mobile environment," in IEEE Veh. Technol. Conf., Chicago, IL, July 1995, vol. 2, pp. 820-824.

[13] L. Rugini and P. Banelli, "BER of OFDM systems impaired by carrier frequency offset in multipath fading Channels," IEEE Trans. Wireless. Commun., vol. 4, pp. 2279-2288, Sept. 2005.

[14] T. Keller and L. Hanzo, "Adaptive multicarrier modulation: A convenient framework for time-frequency processing in wireless communications," in Proc. IEEE, vol. 88, pp. 611-640, May 2000.

[15] K. Sathananthan and C. Tellambura, "Probability of error calculation of OFDM systems with frequency offset," IEEE Trans. Commun., vol. 49, pp. 1884-1888, Nov. 2001.

[16] K. D. P. Dharmawansa, R. M. A. P. Rajatheva and H. Minn, "An exact error probability analysis of OFDM systems with frequency offset," IEEE MILCOM 2006.

[17] K. D. P. Dharmawansa, R. M. A. P. Rajatheva and H. Minn, "An exact error probability analysis of OFDM systems with frequency offset," accepted in IEEE Trans. Commun. 2007. [Online] Available: http://www.utdallas.edu/ Hlaing.Minn/BERfixedCFO.pdf

[18] X. Ma, H. Kobayashi and S. C. Schwartz, "Effect of frequency offset on BER of OFDM and single carrier systems," Proc. IEEE PIMRC 2003, Beijing, China pp. 2339-2243, Sept. 2003.

[19] J. Lee, H.-L. Lou, D. Toumpakaris and J. M. Cioffi, "SNR analysis of OFDM systems in the presence of carrier frequency offset for fading channels," IEEE Trans. Wireless. Commun., vol. 5, pp. 3360-3364, Dec. 2006.

[20] L. Pan and Y.-Bar-Ness, "Comparing the effect of carrier frequency offseton OFDM and single-arrier block transmission in AWGN channels," IEEE GLOBECOM 2006, pp. 1-5, Nov. 2006.

[21] S. Y. Park and C.G. Kang, "Performance of pilot-assisted channel estimation for OFDM system under time-varying multi-path Rayleigh fading with frequency offset compensation," IEEE Vehi. Techno. Conf., vol. 2, pp. 1245-1249, May. 2000.

[22] S. Y. Park, B. S. Seo and C. G. Kang, "Effects of frequency offset compensation error on channel estimation for OFDM system under mobile radio channels," Signal Processing, Elsevier Science, vol. 83, no. 12, pp. 2621-2630, Dec. 2003.

[23] T. Pollet, M. Bladel, and M. Moeneclaey, "BER sensitivity of OFDM systems to carrier frequency offset and Wiener phase noise," IEEE Trans. Commun., vol. 43, pp. 191-193, Feb. 1995.

[24] L. Tomba, "On the effect of wiener phase noise in OFDM systems," IEEE Trans. Commun., vol. 46, pp. 580-583, May. 1998.

[25] A. G. Armada, "Understanding the effects of phase noise in orthogonal frequency division multiplexing (OFDM)," IEEE Trans. Broadcast., vol. 47, pp. 153-159, June 2001.

[26] I. S. Gradshteyn and I. M. Ryzhik, Table of Integrals, Series, and Products, Cambridge, U. K.: Academic Press, 1980.

[27] S. M. Kay, Fundamentals of Statistical Signal Processing: Estimation Theory, Englewood Cliffs, NJ: Prentice-Hall, 1993.

[28] H. Minn and S. Xing, "An optimal training signal structure for frequency offset estimation," IEEE Trans. Commun., vol. 53, pp. 343-355, Feb. 2005 . 
[29] H. Minn, X. Fu and V. K. Bhargava, "Optimal periodic training signal for frequency offset estimation in frequency-selective fading channels," IEEE Trans. Commun., vol. 54, pp. 1081-1096, June 2006.

[30] H. Minn and N. Al-Dhahir, "Optimal training signals for MIMO OFDM channel estimation," IEEE Trans. Wireless Commun., vol. 5, no. 5, pp. 1158-1168, May 2006.

[31] K. S. Miller, "Complex Gaussian processes," SIAM REVIEW, vol. 11, pp. 544-567, Oct. 1969.

[32] S. Verdu, Multiuser Detection, Cambridge University Press, 1998.

[33] J. G. Proakis, Digital Communications, 3rd ed., McGraw-Hill, 1995.

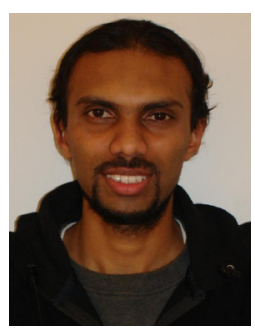

P. C Weeraddana received the B.Sc degree in Electronic and Telecommunication Engineering with first class honors from the university of Moratuwa, Moratuwa, Sri Lanka, in 2004, and the M.Eng degree Telecommunication from School of Engineering and Technology, Asian Institute of Technology, Thailand in 2007. He is currently working toward the Ph.D. degree in Centre for Wireless Communications, Department of Electrical Engineering, University of Oulu, Finland. His research interests are in wireless communications, with a current focus on adaptive radio resource allocation in OFDMA systems, cross-layer optimization and centralized/distributed resource allocation for wireless networks.

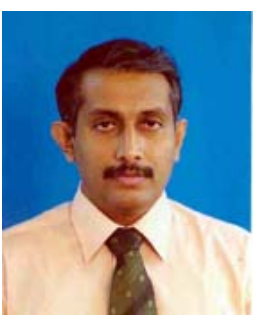

Nandana Rajatheva received the B.Sc. degree in Electronic and Telecommunication Engineering with first class honors from the university of Moratuwa, Moratuwa, Sri Lanka, in 1987, and the M.Sc., Ph.D. degrees from the University of Manitoba, Winnipeg, MB, Canada in 1991 and 1995 respectively.

Currently he is an Associate Professor with the Telecommunications Field of Study, School of Engineeing and Technology, Asian Institute of Technology (AIT), Thailand. He was with the University of Moratuwa, Sri Lanka before joining AIT where he was promoted to Professor in Electronic \& Telecommunication Engineering in June 2003. From May 1996 to Dec.2001, he was with TC-SAT as an Associate Professor. His research interests include mobile and wireless communications, coding and modulation techniques, space time processing for MIMO systems and communication theory.

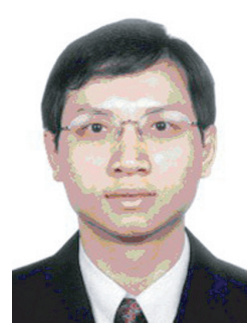

Hlaing Minn (S'99-M'01-SM'07) received his B.E. degree in Electronics from Yangon Institute of Technology, Yangon, Myanmar, in 1995, M.Eng. degree in Telecommunications from Asian Institute of Technology (AIT), Pathumthani, Thailand, in 1997 and Ph.D. degree in Electrical Engineering from the University of Victoria, Victoria, BC, Canada, in 2001.

He was with the Telecommunications Program in AIT as a laboratory supervisor during 1998 . He was a research assistant from 1999 to 2001 and a post-doctoral research fellow during 2002 in the Department of Electrical and Computer Engineering at the University of Victoria. Since September 2002, he has been with the Erik Jonsson School of Engineering and Computer Science, the University of Texas at Dallas, USA, as an Assistant Professor. His research interests include wireless communications, statistical signal processing, error control, detection, estimation, synchronization, signal design, cooperative communication, crosslayer design, and cognitive radios. He is an Editor for the IEEE Transactions on Communications. 